\title{
GEODIVERSIDADE DOS COMPARTIMENTOS GEOMORFOLÓGICOS DO ANTICLINAL DE MARIANA, MINAS GERAIS
}

\author{
GEODIVERSITY OF THE GEOMORPHOLOGICAL COMPARTMENTS OF THE MARIANA \\ ANTICLINAL, MINAS GERAIS STATE, BRAZIL
}

\section{Stênio Toledo NASCIMENTO ${ }^{1}$, Paulo de Tarso Amorim CASTRO ${ }^{1}$, Úrsula de Azevedo RUCHKYS ${ }^{2}$}

${ }^{1}$ UFOP - Universidade Federal de Ouro Preto. Emails: steniotoledo@gmail.com; ptacastro@gmail.com

${ }^{2}$ UFMG - Universidade Federal de Minas Gerais. Email: tularuchkys@yahoo.com.br

\author{
Introdução \\ Área de Estudo \\ Materiais e Métodos \\ Resultados e Discussões \\ Conclusão \\ Agradecimentos \\ Referências
}

\begin{abstract}
RESUMO - Estudos recentes propõem diversos métodos para quantificar a geodiversidade de diferentes regiões do planeta. Neste trabalho foram utilizados dados vetoriais para determinar a geodiversidade múltipla simples e ponderada do Anticlinal de Mariana. O Anticlinal de Mariana está localizado na região sudeste no Quadrilátero Ferrífero, região central do estado de Minas Gerais, entre os municípios de Ouro Preto e Mariana. A quantificação da geodiversidade na região foi realizada de acordo com seis compartimentos definidos segundo as características geológicas, geomorfológicas e estruturais locais. Estes compartimentos foram classificados considerando o grau de geodiversidade, variando de muito baixa a muito alta havendo diferença nos resultados obtidos entre a geodiversidade múltipla simples e múltipla ponderada.
\end{abstract}

Palavras-chave: Geodiversidade, Anticlinal de Mariana, Quantificação, Vetores.

ABSTRACT - Recent studies propose several methods to quantify the geodiversity of different regions of the planet. This work used vector data to determine the multiple geodiversity simple and weighted of the Anticlinal de Mariana. The Mariana Anticlinal is located in southeast of the Quadrilátero Ferrífero, Minas Gerais, between the towns of Ouro Preto and Mariana. Quantification of the geodiversity in the region was performed according to six compartments defined to the geological characteristics, geomorphological and structural local. These compartments were classifieds according with degree of geodiversity, ranging from very low to very high and there was variation in the different methods applied.

Keywords: Geodiversity, Mariana Anticlinal, Quantify, Vector.

\section{INTRODUÇÃO}

O termo geodiversidade começou a ser usado no início dos anos 1990, graças às publicações que discutiam a variedade das características relacionadas aos elementos físicos da natureza. A partir de então tem sido objeto de estudos em várias áreas do conhecimento, notadamente na geologia, geografia, turismo e biologia. Nessas áreas a geodiversidade vem se consolidando em termos conceituais e metodológicos. Fontana et al. (2015) salientam que essa consolidação se deve, em parte, ao interesse crescente pelos temas da Terra em um cenário atual de degradações ambientais em escala planetária.

$\mathrm{O}$ termo geodiversidade foi definido por diversos autores como Alexandrowicz \& Kozlowski (1999), Xavier-da-Silva (2001); Stanley (2001); Brilha, (2005), dentre outros, com abordagens variadas em relação aos elementos que o constituem e sua importância e valores patrimoniais associados.

Gray (2004), geógrafo inglês, tem sido uma referência mundial nos estudos de geodiver- sidade. Para ele a geodiversidade se refere à variedade de elementos geológicos, geomorfológicos, pedológicos e hidrográficos que contribuem para a estruturação da paisagem.

Essa variedade do meio abiótico pode ser analisada tanto do ponto de vista quantitativo como qualitativo. As pesquisas envolvendo análise qualitativa da geodiversidade partem de uma descrição de valores a ela associados e são mais difundidas. Já aqueles trabalhos que têm como objetivo avaliar e gerar modelos de geodiversidade, buscando mostrar sua integração com a paisagem e com os processos formadores desta, são menos comuns. Manosso \& Ondicol (2012) salientam que um dos principais objetivos de avaliar a geodiversidade e sua distribuição espacial é subsidiar a geoconservação. Para Ferreira (2014) além de promover um conhecimento do patrimônio geológico da área, a quantificação da geodiversidade também pode contribuir para planejamentos ambientais e de uso e ocupação do solo. 
Segundo Manosso (2012) na análise quantitativa alguns trabalhos partem do processo de compartimentação geomorfológica, realizado a partir das estruturas, formas, processos e clima, que são uma primeira maneira de se chegar à geodiversidade de uma área. De fato a morfologia do terreno é determinada pela interação de processos naturais endógenos e exógenos e um mapa de compartimentação geomorfológica sintetiza, de certa forma, essa interação.

Serrano \& Ruiz-Flaño (2007) realizaram uma compartimentação geomorfológica na região de Caracena, província de Sória, na Espanha e calcularam índices de geodiversidade para cada um dos compartimentos mapeados. Essa abordagem permite uma interpretação de forma integrada, pois a geodiversidade e a fisionomia da paisagem são resultantes das mudanças geomorfológicas vigentes e pretéritas.

O método desenvolvido se baseia em modelos de riqueza, densidade e distribuição de índices de diversidade. O cálculo é realizado de acordo com os compartimentos geomorfológicos mapeados para os quais são designados valores de geodiversidade conforme a variedade de elementos físicos em cada unidade.

Segundo Pereira et al. (2012), o uso de metodologias para avaliação quantitativa e distribuição espacial da geodiversidade é muito recente e se baseia, principalmente, na sobreposição cartográfica em Sistema de Informação Geográfica (SIG) de elementos do meio físico, resultando em um mapa de geodiversidade. Os mapas de geodiversidade podem ser usados como subsídio ao planejamento territorial, à conservação da natureza, à gestão dos recursos naturais e ao uso turístico do território.

Nesse contexto, o objetivo deste trabalho é mapear os compartimentos geomorfológicos do Anticlinal de Mariana e quantificar a geodiversidade de cada uma das unidades mapeadas considerando a geodiversidade múltipla simples e a geodiversidade múltipla ponderada. Segundo Xavier da Silva et al. (2001), o índice de geodiversidade múltipla simples é obtido pela soma das classes encontradas em cada plano de informação do meio físico utilizado. Já o índice de geodiversidade múltipla ponderada é obtido dividindo-se o número de classes encontradas em cada plano de informação pela sua área total de ocorrência.

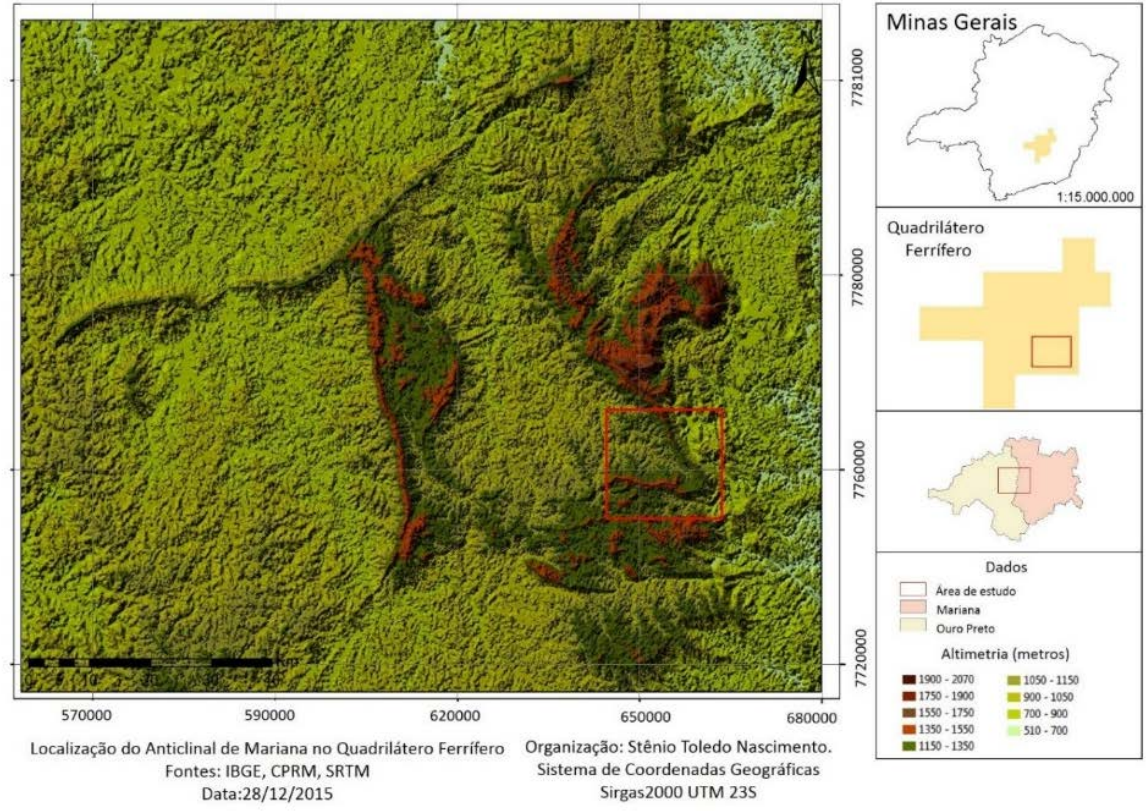

Figura 1 - Localização da área de estudo no Quadrilátero Ferrífero, entre os municípios de Ouro Preto e Mariana.

\section{ÁREA DE ESTUDO}

O Anticlinal de Mariana é uma estrutura regional da porção sudeste do Quadrilátero Ferrífero, área clássica da geologia e da mineração de Minas Gerais. A Serra de Ouro
Preto está no flanco sul do anticlinal, a serra de Antônio Pereira no flanco nordeste e a Passagem de Mariana na zona de charneira deste Anticlinal (Figura 1). 
Segundo Alkmim \& Marshak (1998), o Quadrilátero Ferrífero apresenta, litoestratigraficamente (Figura 2), os seguintes conjuntos rochosos: Complexos Metamórficos que formam embasamento cristalino arqueano (Belo Horizonte, Caeté, Bomfim, Santa Bárbara e Bação); o Supergrupo Rio das Velhas; o Supergrupo Minas; as intrusivas do Supergrupo Minas e o Grupo Itacolomi.

O Anticlinal de Mariana é formado por rochas arqueanas do Supergrupo Rio das Velhas e proterozóicas do Supergrupo Minas, que se encontram justapostas (Ladeira 1984, 1988). Essa estrutura regional do Quadrilátero Ferrífero apresenta grande valor patrimonial do ponto de vista da história da mineração e do patrimônio geológico. Nalini Júnior et al.
(1992) salientam que o Anticlinal de Mariana despertou o interesse de Eschwege (1818) pela ocorrência de mineralizações auríferas assim como o de Gorceix (1881) que estudou sua estruturação. Mais recentemente esse valor patrimonial é objeto de estudo de Fonseca et al. (2001), que tratam do sítio arqueológico do Morro da Queimada; de Sobreira (2014), que relaciona às mudanças ocorridas na paisagem do Anticlinal de Mariana à mineração de ouro nos últimos três séculos. A Serra de Antônio Pereira, flanco nordeste do anticlinal, tem seu valor espeleológico salientado por Munaro (2011) que discorre sobre a presença de cavidades naturais e antrópicas próximas aos sítios arqueológicos Morro Santana e Morro Santo Antônio.

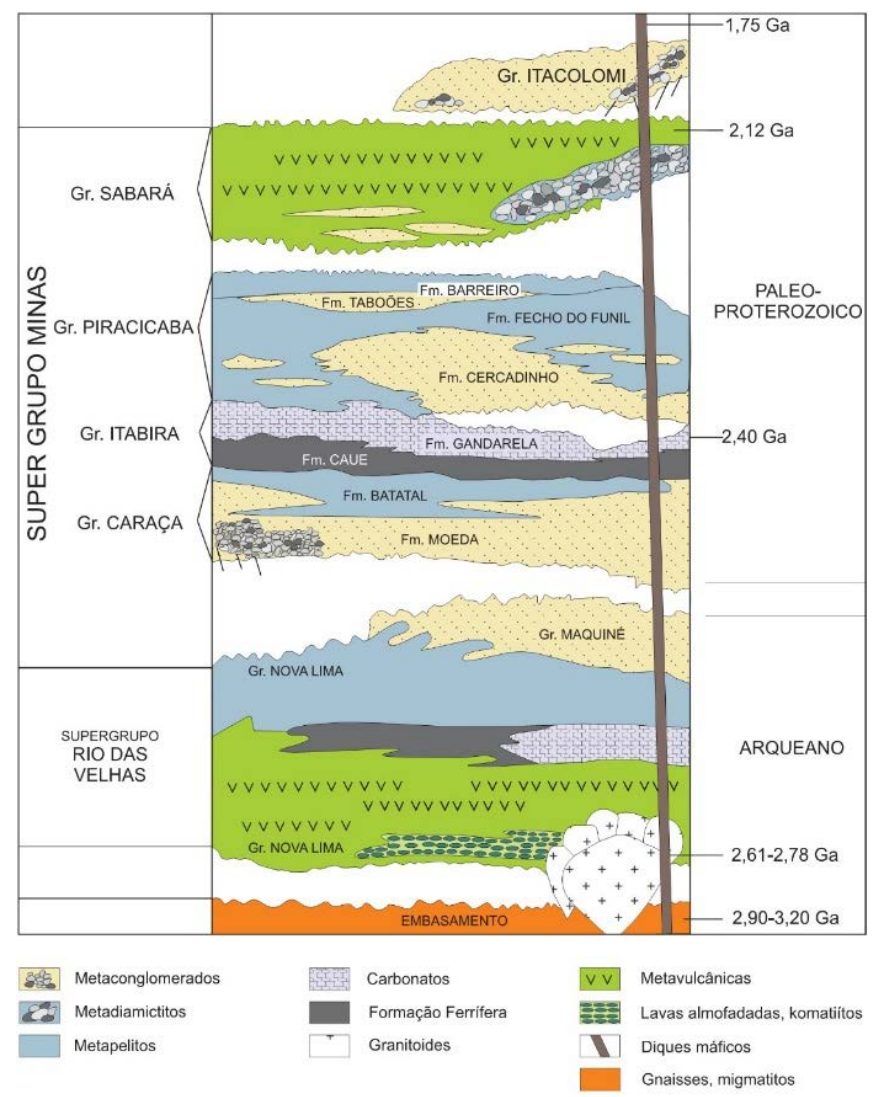

Figura 2 - Coluna estratigráfica do Quadrilátero Ferrífero. Adaptado de Alkmim e Marshak (1998).

\section{MATERIAIS E METODOS}

As bases cartográficas envolvendo a geologia e as unidades hidrogeológicas, em formato digital, foram obtidas do projeto "Geologia do Quadrilátero Ferrífero Integração e Correção Cartográfica em SIG”, resultante da compilação dos dados realizada pela Companhia de Desenvolvendo Econômico de Minas Gerais - CODEMIG (2005) em escala 1:25.000 seguindo articulação 1:50000 do
IBGE. Os estudos geomorfológicos utilizaram como base imagens de radar e satélite com auxílio do software Google Earth Pro e os dados topográficos digitais SRTM com resolução em pixels de 1 arc-second, aproximadamente $30 \mathrm{~m}$, de livre acesso no site do Serviço Geológico dos Estados Unidos (U.S.G.S. - United States of Geological Survey). As ocorrências minerais foram obtidas 
do banco de dados do Serviço Geológico Brasileiro (CPRM), no site http://geobank.cprm. gov.br/, no qual é possível se obter a shapefile da área desejada em livre acesso.

A primeira etapa do método envolveu a compartimentação geomorfológica do Anticlinal de Mariana. A compartimentação se baseou nos diferentes níveis topográficos e características litológicas da região que sintetizam os aspectos estruturadores das formas de relevo. Após uma primeira etapa de campo de reconhecimento foi criado um Modelo Digital de Elevação (MDE) e gerados mapas de hipsometria, declividade, relevo sombreado e perfis topográficos. Com uso do software ArcGis esses mapas e os perfis foram analisados de forma combinada com a geologia da área o que permitiu a elaboração do mapa de compartimentação com definição de unidades com características geológicoestruturais semelhantes. Para validação do mapa gerado foram realizadas novas atividades de campo onde cada unidade geomorfológica mapeada foi caracterizada.

A segunda etapa envolveu a quantificação da geodiversidade do Anticlinal de Mariana. Inicialmente foram selecionadas as variáveis que seriam utilizadas: geologia, hidrografia, geomorfologia, ocorrência mineral. Estas variáveis foram escolhidas devido à disponibilidade dos dados, a relevância em relação à área de estudo e uniformidade da escala de trabalho (1:50.000). A Tabela 1 sintetiza as classes usadas de cada uma das variáveis com seus respectivos números.

Tabela 1: Variáveis x Número de Classes.

\begin{tabular}{ccc}
\hline \multirow{2}{*}{ Variáveis } & Classes & Número de classes \\
\hline \multirow{3}{*}{ Geologia } & Litologia & 32 \\
\cline { 2 - 3 } & Idades Geológicas & 7 \\
\cline { 2 - 3 } & Formação Geológica & 16 \\
\cline { 2 - 3 } Geomorfologia & Unidades Geomorfológicas & 5 \\
\cline { 2 - 3 } & Declividade & \multicolumn{1}{c}{6} \\
\cline { 2 - 3 } & Rugosidade & 10 \\
\hline Hidrografia & Orientação da vertente & \multicolumn{1}{c}{5} \\
\hline Ocorrência mineral & Ocorrência Mineral & $\mathbf{1 1 5}$ \\
\hline & Total de elementos & \\
\hline
\end{tabular}

A quantificação da geodiversidade foi realizada com aplicação do método proposto por Xavier da Silva et al. (2001), usado por Dias (2005), Grigio (2011) e Manosso (2012). As classes de geodiversidade representam a variabilidade encontrada na área de estudo, que foram analisadas pela Geodiversidade Múltipla Simples e Geodiversidade Múltipla Ponderada.

A Geodiversidade Múltipla Simples (GMS) é a soma das classes encontradas em cada mapa temático básico, enquanto a Geodiversidade
Múltipla Ponderada (GMP) é obtida dividindose o número de classes encontradas em cada classe padrão pela sua área total de ocorrência. São identificados e avaliados os elementos que compõem a geodiversidade associados aos aspectos geológicos, geomorfológicos, hidrológicos e à ocorrência mineral. Esse método permite a análise em diferentes escalas, desde elementos da geodiversidade observados em nível de paisagem até aqueles observados em nível de afloramento.

\section{RESULTADOS E DISCUSSÕES}

A área de estudo foi segmentada em seis compartimentos (Figura 3). Observa-se que o Anticlinal de Mariana possui uma paisagem bastante diversificada, com características associadas às inúmeras combinações da estrutura geológica e geomorfológica existentes.

O compartimento 1 (C1) indica o flanco sul do Anticlinal de Mariana, enquanto o compartimento 2 (C2) representa o flanco nordeste, formando as bordas deste Anticlinal. A zona de charneira do Anticlinal é representada pelo compartimento 3 (C3), porção sudeste da área de estudo. O compartimento 4 (C4) é a delimitação do vale 
do Rio das Velhas. O compartimento 5 (C5) apresenta um relevo de planalto onde está situada a sede do município de Mariana. O compartimento 6 (C6) indica a transição dos domínios Quadrilátero Ferrífero e Planaltos Dissecados (RADAMBRASIL, 1983).

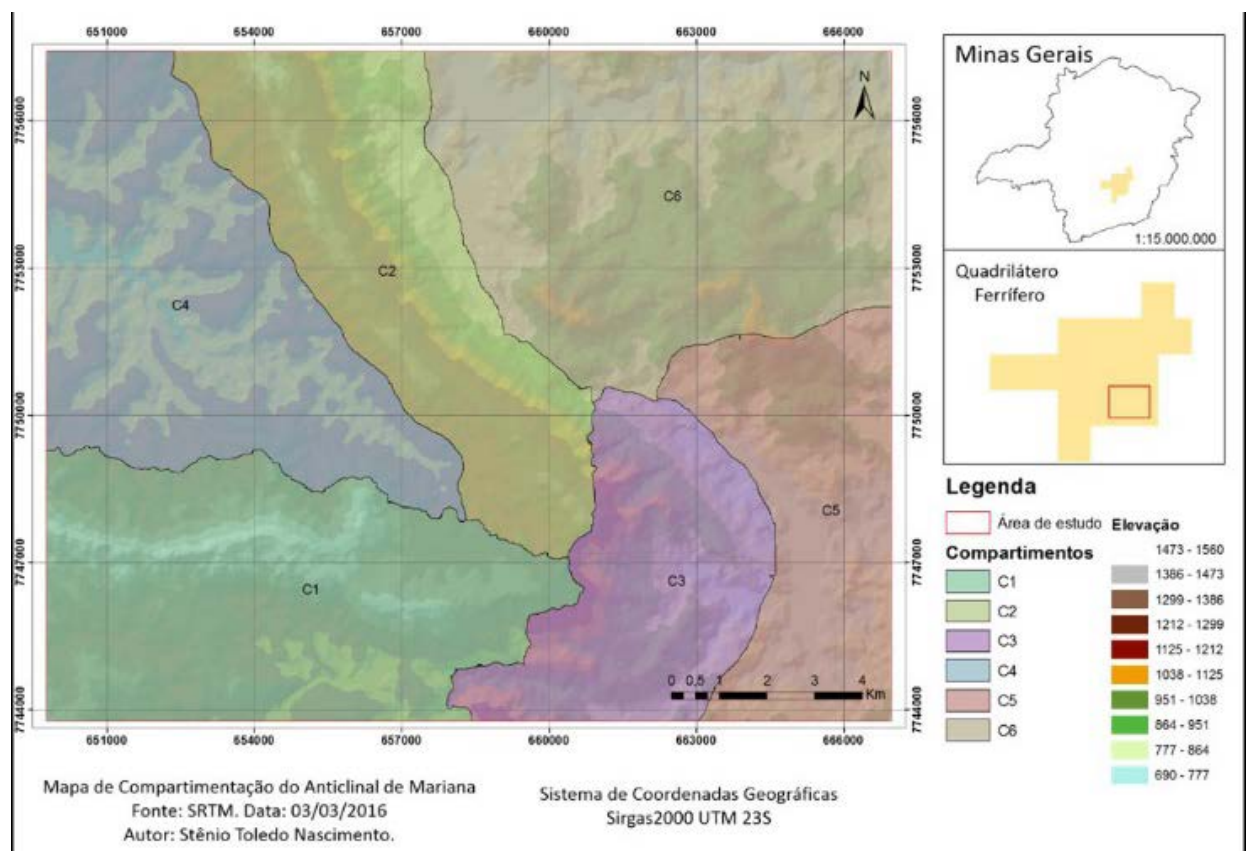

Figura 3 - Mapa de compartimentação do Anticlinal de Mariana.

A quantificação da geodiversidade é resultado das operações matemáticas em que são analisados diferentes elementos de cada variável que aparecem nos compartimentos determinados anteriormente. Foram analisados 115 elementos que aparecem distribuídos em uma área de aproximadamente $235 \mathrm{~km}^{2}$.

$\mathrm{O}$ resultado da quantificação da geodiversi- dade aparece na tabela 2, que traz o cálculo da geodiversidade múltipla simples (quantidade de elementos por compartimentos), das áreas em quilômetros quadrados por compartimento, e da geodiversidade múltipla ponderada. $\mathrm{O}$ cruzamento de dados envolveu as variáveis Geologia, Geomorfologia, Hidrografia e Ocorrência Mineral.

Tabela 2: Quantificação da geodiversidade por compartimentos.

\begin{tabular}{cccc}
\hline Compartimentos & GMS $^{\mathbf{1}}$ & Área $\mathbf{( K m}^{\mathbf{2}} \mathbf{~}$ & $\mathbf{G M P}^{\mathbf{2}}$ \\
\hline $\mathbf{C 1}$ & 80 & 47 & 1,702 \\
\hline $\mathbf{C} 2$ & 79 & 37 & 2,135 \\
\hline C3 & 70 & 25 & 2,800 \\
\hline C4 & 62 & 45 & 1,377 \\
\hline C5 & 74 & 26 & 2,846 \\
\hline C6 & 76 & 54 & 1,407
\end{tabular}

${ }^{1}$ GMS: Geodiversidade múltipla simples;

${ }^{2}$ GMP: Geodiversidade Múltipla Ponderada.

Para qualificação da Geodiversidade Múltipla Simples e Geodiversidade Múltipla Ponderada em níveis que vão de Muito Baixa a Muito Alta, foi realizado um estudo de dispersão de valores das colunas GMS e GMP da tabela 2, observando o desvio padrão calculado por ferramenta própria do software ArcGis. No desvio padrão, é possível avaliar o quanto o valor de atributo da feição varia a partir da média dos dados, permitindo assim a criação de classes a partir dos índices de geodiversidade calculados.
A figura 4 apresenta o mapa de Geodiversidade Múltipla Simples e mostra que os compartimentos C1 e C2, flanco sul e nordeste do Anticlinal de Mariana são aqueles com o maior número de elementos da geodiversidade, 80 e 79, respectivamente. $\mathrm{Na}$ análise geomorfológica local, são caracterezados predominantemente por topos de morros e escarpas. Foi atribuída, então, a classificação Geodiversidade Alta para esses compartimentos. 


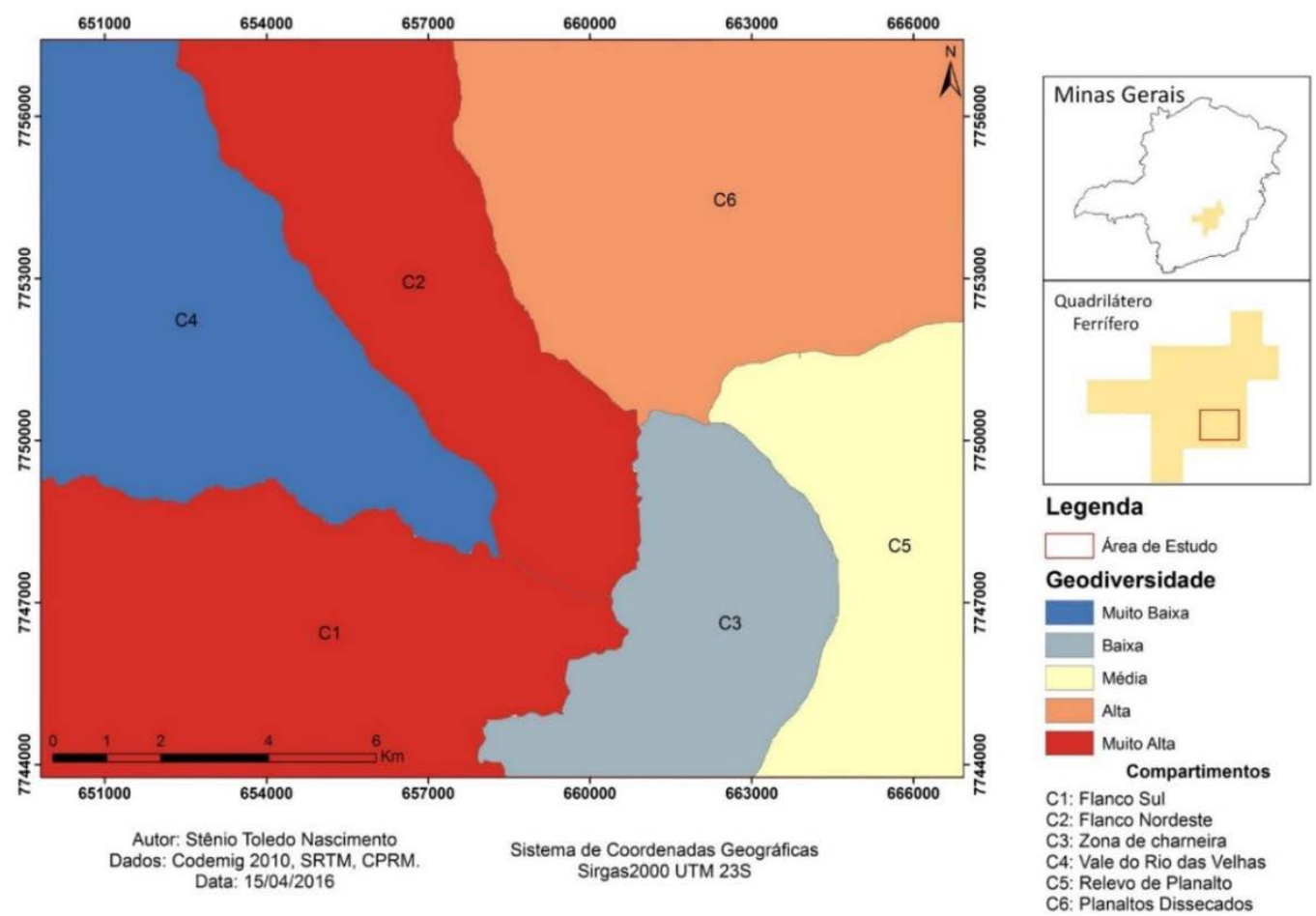

Figura 4: Geodiversidade Múltipla Simples do Anticlinal de Mariana.

O compartimento C6 apresenta geodiversidade alta, com 76 elementos distintos. Este compartimento representa a transição entre o Quadrilátero Ferrífero e os Planaltos Dissecados, com relevos ondulados, planaltos e morros com topos arredondados. O compartimento C5, relevo de planalto onde se localiza a cidade de Mariana, apresenta geodiversidade média, com 74 elementos das variáveis utilizadas neste estudo.

O mapa apresentado na figura 5 corresponde ao mapa de Geodiversidade Múltipla Ponderada, ou seja, os valores encontrados são resultantes da distribuição da Geodiversidade Múltipla Simples levando em consideração as áreas de cada compartimento.

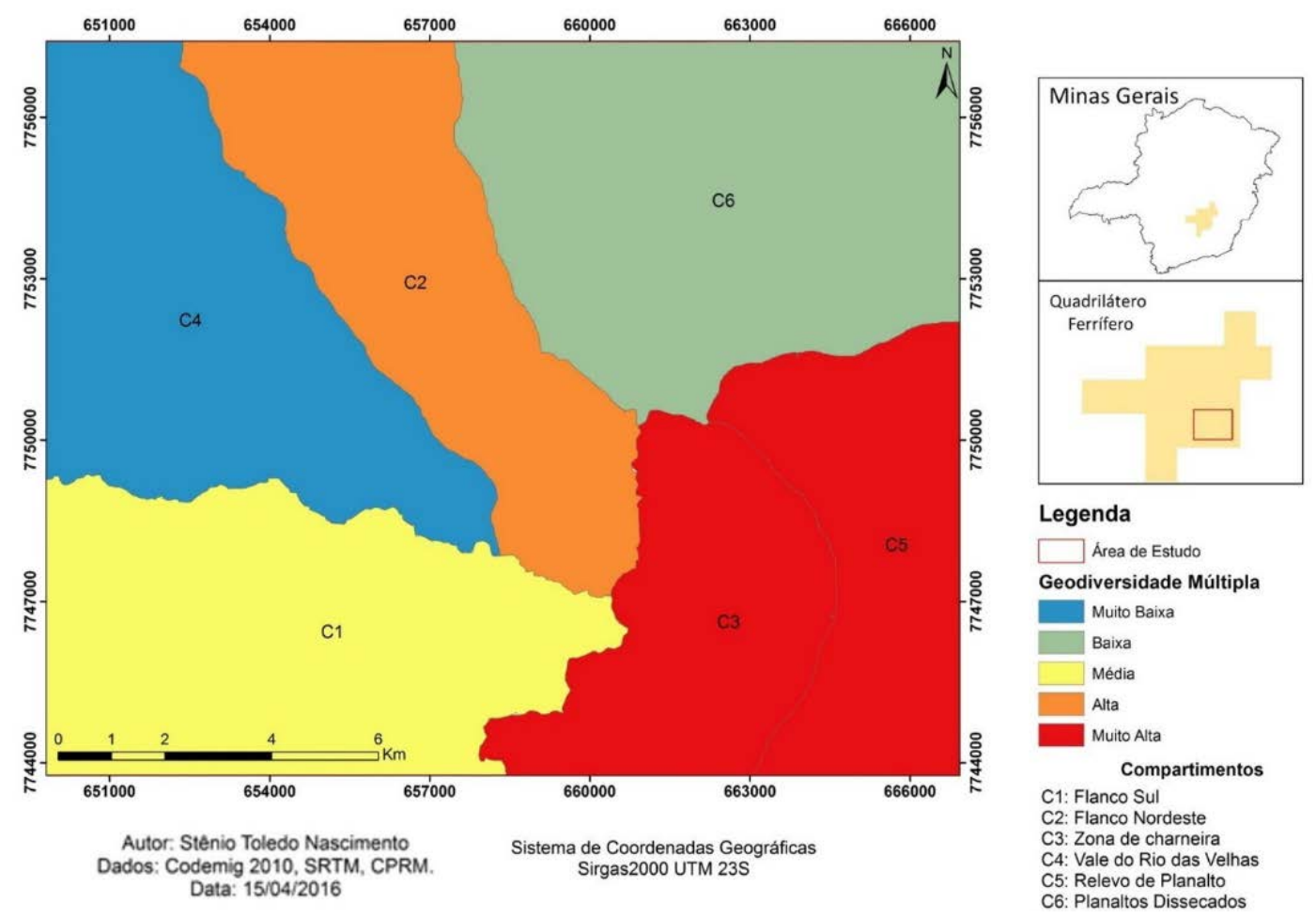

Figura 5: Geodiversidade Múltipla Ponderada do Anticlinal de Mariana. 
$\mathrm{Na}$ análise de Geodiversidade Múltipla Ponderada se observa que tanto o compartimento C3, zona de charneira, quanto o compartimento C5, relevo de planalto, apresentam geodiversidade muito alta. Isto ocorre porque as áreas destes compartimentos são menores que as dos demais, entretanto, o número de elementos, GMS, variam numa faixa de valores pequena, aumentando o grau de geodiversidade por quilômetro quadrado.

No sentido inverso, o compartimento C6 no GMS apresenta geodiversidade alta, enquanto a GMP apresenta índices baixos de geodiversidade devido à extensa distribuição dos elementos geológicos no maior compartimento do Anticlinal de Mariana.

Os flancos do Anticlinal de Mariana, compartimentos C1 e C2, aparecem com geodiversidade de média a alta na GMP, embora na GMS apresentem os maiores valores. O compartimento $\mathrm{C} 4$, referente ao vale do rio das Velhas, mantém a classificação da GMS e aparece com geodiversidade muito baixa na GMP.

Os resultados encontrados mostram que há diferenças entre padrões de geodiversidade no Anticlinal de Mariana quando se considera a distribuição espacial dos elementos geológicos e geomorfológicos utilizados no cálculo do índice de geodiversidade, tanto na GPS quanto na GPM, e as áreas dos compartimentos da região de estudo. Assim como pode ser observado em casos em que houve a inversão, ou seja, compartimentos com classificações altas de geodiversidade na GMS passam a ser classificados como baixas na GMP e vice-versa.

\section{CONCLUSÃO}

A metodologia aplicada e os resultados obtidos mostraram-se eficazes no cálculo do índice de geodiversidade para o Anticlinal de Mariana. Devido às características do Quadrilátero Ferrífero, em que elementos geológicos e geomorfológicos são evidentes e variam muito em pequenas áreas, foi possível constatar que existe um padrão de altos valores de índice geodiversidade quando se trata da distribuição espacial dos elementos estudados da área como um todo.

Foram apresentados os resultados da quantificação da geodiversidade múltipla simples e ponderada por compartimentos do Anticlinal de Mariana utilizando dados vetoriais de elementos da geodiversidade, permitindo observar a influência das áreas destes compartimentos no momento de classificar a geodiversidade na região estudada.
Vale destacar que os estudos de geodiversidade são guias para outros estudos que envolvem o meio físico. Sendo assim, diferentes métodos de quantificação da geodiversidade podem ser adequados a depender dos objetivos específicos de cada trabalho. A questão da disponibilização de dados em escala de detalhe é fundamental para esse tipo de estudo e pode ter uma grande influência no resultado final. Seria importante que, por sua importância paisagística e geológica, o Anticlinal de Mariana tivesse seu mapeamento em escalas maiores como 1:25.000 considerando os diferentes elementos que compõem sua diversidade física. Espera-se que esse estudo possa servir como subsídio ao planejamento do uso da área priorizando ações de conservação para aqueles compartimentos classificados como de mais alta geodiversidade.

\section{AGRADECIMENTOS}

Agradecimento ao Programa de Pós-Graduação em Evolução Crustal e Recursos Naturais Departamento de Geologia da Escola de Minas - UFOP, Programa de Pós-Graduação em Análise e Modelagem de Sistemas Ambientais - Universidade Federal de Minas Gerais e à Coordenação de Aperfeiçoamento de Pessoal de Nível Superior.

\section{REFERÊNCIAS}

ALEXANDROWICKZ, Z. \& KOZLOWSKI, S. From selected geosites to geodiversity conservation. Polish example of modern framework. In: Barettion D. M. (eds.) Towards the balanced management and conservation of the Geological Heritage in the New Milenium. Sociedade Geológica da España, Madrid, p.: 40-44. 1999.

ALKMIM F.F. \& MARSHAK S. Transamazonian Orogeny in the Southern São Francisco Craton Region, Minas Gerais,
Brazil: evidence for Paleoproterozoic collision and collapse in the Quadrilátero Ferrífero. Precambrian Research, n. 90, p.29-58, 1998.

BRILHA J. Patrimônio Geológico e Geoconservação: a conservação da natureza na sua vertente geológica. Portugal, Palimage, 190p. 2005.

DIAS, J.D.; GOMES, O.V.O.; XAVIER DA SILVA, J.; GOES, M.H.B. A geodiversidade do município de Volta Redonda, 
Rio de Janeiro. Caminhos da Geografia, n. 14, v. 14, pgs. 151-160, 2005.

FERREIRA, B. (2014). Geodiversidade do Estado de Pernambuco, NE do Brasil. Tese do doutorado. Departamento de Geociências. Universidade Federal de Pernambuco. 254 pp. Recife, Pernambuco.

FONSECA, M.; SOBREIRA, F.; ESTELA RAINHO, M.; OLIVEIRA, M. The Morro da Queimada Archaeological Site, Ouro Preto, Brasil. Cities, Vol 18, No 6, pp. 381-289. 2001.

FONTANA, R.C., MENEGAT, R.; MIZUSAKI, A.M.P. Geoconservação em Grandes Cidades e Proposição dos Itinerários Geológicos de Porto Alegre: Contribuições Metodológicas para Valoração Integrada de Unidades Geológicas. São Paulo, UNESP, Geociências, v. 34, n. 4, p.897-918, 2015

GRAY, M. Geodiversity: valuing and conserving abiotic nature. Chichester: Wiley, 434 p., 2004.

GRIGIO, A.M.; AMARO, V.E.; DIODATO, M.A.; CASTRO, A.F.. Determination of indices of multiple and Multiple Weighted Geodiversity of landscape of the Piranhas-Assu river, Rio Grande do Norte - Brazil. Journal of Coastal Research, SI 64 (Proceedings of the 11th International Coastal Symposium), p. 1668 - 1671. Szczecin, Poland. 2011.

LADEIRA E.A. Metalogenia dos depósitos de ouro do Quadrilátero Ferrífero, Minas Gerais. In: Schobbenhaus, C.; Coelho, C. E. S. Principais Depósitos Minerais do Brasil. Brasília: DNPM, 1988. v. 3, p. 301-375.

MANOSSO C.F. \& ONDICOL R.M. Geodiversidade: Considerações Sobre Quantificação e Avaliação da Distribuição Espacial. Anuário do Instituto de Geociências, 35: 90 - 100. 2012.

MANOSSO, F. C. Potencialidades da paisagem na Região da Serra do Cadeado-PR: abordagem metodológica das relações entre a estrutura geoecológica, a geodiversidade e o geoturismo. Tese de Doutorado, Centro de Ciências Humanas, Letras e Artes, Universidade Estadual de Maringá, 183 p. 2012. Maringá, Paraná.

MUNARO, P. Estudo Espeleológico Comparativo Em Cavidades Naturais Subterrâneas, Impactadas E Antrópicas Em Formações Ferríferas. In: ANAIS do $31^{\circ}$ Congresso Brasileiro de Espeleologia - Ponta Grossa-PR - Sociedade Brasileira de Espeleologia. 2011.

NALINI JÚNIOR, H.A.; ROSIÈRE, C.A.; ENDO, I. Sobre a geologia estrutural do anticlinal de Mariana, região sudeste do Quadrilátero Ferrífero Minas Gerais: uma revisão. Revista Escola de Minas, Ouro Preto, v. 45 1/2, p. 18-20, 1992.
PEREIRA D.; SANTOS L.; SILVA J.; PEREIRA P.; BRILHA J.; FRANÇA J.; RODRIGUES C. Finding the proper methodology for geodiversity assessment: a recent approach in Brazil and Portugal. European Geosciences Union Geophysical Research Abstracts, 2012. Vol. 14, EGU General Assembly - Viena.

RADAMBRASIL. Folhas SF 23/24, Rio de Janeiro/Vitória. Geologia, geomorfologia, pedologia, vegetação e uso potencial da terra. 780p. 1983.

SERRANO E. \& RUIZ-FLAÑO P. Geodiversidad: concepto, evaluación y aplicación terrotorial. El caso de Tiermes Caracena (Soria). Boletín de la A.G.E. 45:79-98. 2007.

SOBREIRA, F. Mineração do ouro no período colonial: alterações paisagísticas antrópicas na serra de Ouro Preto, Minas Gerais. 2014. Quaternary and Environmental Geosciences, v. 5, n. 1. Disponível em <revistas.ufpr.br/ abequa/article/download/34432/23233> Acesso em 28 jun. 2016.

STANLEY M. Geodiversity strategy. Progeo News 1:6-9. 2001.

XAVIER-DA-SILVA, J. Geoprocessamento para análise ambiental. Rio de Janeiro, 228 pp. (Eds.) 2001.

XAVIER-DA-SILVA, J.; PERSON, V.G., LORINI, M.L., BERGAMO, R.B.A.; RIBEIRO, M.F.; COSTA, A.J.S.T.; IERVOLINO, P.; ABDO, O.E. Índices de geodiversidade: aplicações de SIG em estudos de biodiversidade. In: GARAY, I.; DIAS, B.F.S. (Orgs.). Conservação da biodiversidade em ecossistemas tropicais: avanços conceituais e revisão novas metodologias de avaliação e monitoramento. Rio de Janeiro: Vozes, 2001. p. 299-316. 